\title{
Perspectives on Personalized Nutrition for Obesity
}

\author{
J. Alfredo Martínez \\ CIBERobn, Physiopathology of Obesity and Nutrition, Institute of Health Carlos III, \\ Madrid, and Department of Nutrition, Food Science and Physiology, University of \\ Navarra, Pamplona, Spain
}

Obesity can result from the disequilibrium between energy intake and expenditure associated with alterations of many metabolic pathways [1]. As many cellular functions related to energy homeostasis are regulated by gene expression and gene-environment interactions, individual variation in body weight/composition and adipose metabolism could be influenced by genetic makeup and nutrient intake. Nutritional outcomes can also be determined by gene-mediated biochemical pathways that regulate nutrient absorption, distribution, metabolism, and excretion as well as other cellular energy processes [2]. Indeed, the interplay between nutrients and genetic variation could be a key factor influencing obesity risk and development as well as associated conditions.

About $25-70 \%$ of body weight variability is thought to be controlled by genes that affect energy homeostasis through the modulation of nuclear transcription factors, signalling molecules and receptors, adipogenesis and fat deposition, thermogenesis, hypothalamic networks, and other cellular functions related to energy efficiency [3, 4]. More than 50 genes have been implicated in obesity phenotypes, and adiposity traits have been identified in both animal and human studies [5]. For example, monogenic obesity is attributed to the mutation of specific alleles with profound effects, namely in the leptin, leptin receptor, pro-opiomelanocortin, proprotein convertase/kexin type 1, melanocortin-4 receptor, and single-minded homolog 1 genes. However, monogenic obesity is relatively uncommon, and studies on polygenic obesity support the notion that the modern prevalence of obesity has resulted from the interactions of unfavourable lifestyles with specific gene variants or single-nucleotide polymorphisms (SNPs) with less profound but converging effects [6]. Individuals susceptible to excessive fat gain may therefore carry gene variants that influence appetite control (CNR1, NPY, POMC, MC4R, etc.), nuclear and cytoplasm regulatory machinery (FTO, TFAPB2, TCF7L2, SCAP, DRD2, etc.), adipogenesis and lipid metabolism (ADRB3, PPAR, APOs, PLIN, etc.), energy expenditure (UCPs), insulin signalling (ISR-2, INSIG2, GIPR), and inflammation (ADIPOQ, IL-6, RESISTIN, etc.) [6]. Over 500 SNPs or chromosomal regions have been directly or indirectly associated with obesity [7].

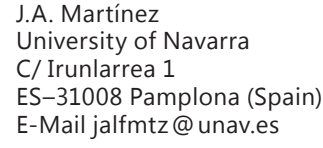


In this context, the field of nutrigenetics offers novel tools to elucidate the roles of obesityrelated genes and to understand how we may modulate them through personalized nutrition. Advances in nutrigenetics are based on the understanding that the genetic makeup determines unique nutritional requirements and rely on the sequencing of the human genome and subsequent analyses of human genetic variation as well as studies that associate gene variants with diseases [5]. By taking into account the diversity of the inherited genome together with the myriad interactions of genes with specific foods or nutrients, nutrigenetics knowledge contributes to the development of personalized nutrition [8]. Thus, it is not only important to characterize the influence of an individual's genetic background on the onset of obesity [9], but also to consider the method by which nutritional interventions should be prescribed on an individual basis.

The management of obesity requires understanding of the genetic and environmental contributors to an individual's health status, including the identification of specific polymorphisms and interpretation of responses to nutritional intervention that may be affected by genetic determinants [10]. For example, subjects carrying obesity-related SNPs have shown differences in response to caloric restriction programmes and may also be differentially affected by the composition of their dietary intake $[11,12]$.

Recent efforts by international consortia aim to describe genetic variants that contribute to obesity in order to characterize nutrient-gene interactions and to implement dietary counselling for obesity prevention and management in a tailored manner [10]. Since genotyping and gene expression appraisal on nutrient-sensitive candidate genes contribute to advancements in obesity diagnosis, prognosis, and therapy, additional nutrigenetics and nutrigenomics studies are needed to support the clinical prescription of personalized nutrition interventions [13].

There remains debate over whether personalized nutrition for obesity is ready for implementation in terms of acceptance, privacy protection, marketing delivery and distribution, affordability, cost, and reliability aspects. Direct-to-consumer genetic testing is already being used in various countries to implement individualized treatments based on genotype and is gaining utility in weight management [14]. However, studies reveal inconsistencies in the interpretation of and disparities concerning risk assessment [15] as well as difficulties in perceptions concerning the application of direct-to-consumer genetic tests or the estimated lifetime risk values, since uncertainties still exist [12]. The application of direct-to-consumer genome-wide profiling to assess disease risk and for therapy purposes requires further research concerning the use, description, implementation, and impact, where methodological and ethical questions need to be accounted for [12]. Other methods for implementing customized nutrition approaches therefore warrant consideration; for example, professional consultation incorporating genetic information related to obesity risk and development may be helpful for the prevention or management of obesity in individuals.

\section{References}

1 Milagro FI, Mansego ML, De Miguel C, Martínez JA: Dietary factors, epigenetic modifications and obesity outcomes: progresses and perspectives. Mol Aspects Med 2013;34:782-812.

2 Jenab M, Slimani N, Bictash M, Ferrari P, Bingham SA: Biomarkers in nutritional epidemiology: applications, needs and new horizons. Hum Genet 2009;125:507-525.

3 Day FR, Loos RJ: Developments in obesity genetics in the era of genome-wide association studies. J Nutrigenet Nutrigenomics 2011;4:222-238.

4 El-Sayed Moustafa JS, Froguel P: From obesity genetics to the future of personalized obesity therapy. Nat Rev Endocrinol 2013;9:402-413. 
5 Fenech M, El-Sohemy A, Cahill L, Ferguson LR, French TA, Tai ES, Milner J, Koh WP, Xie L, Zucker M, Buckley M, Cosgrove L, Lockett T, Fung KY, Head R: Nutrigenetics and nutrigenomics: viewpoints on the current status and applications in nutrition research and practice. J Nutrigenet Nutrigenomics 2011;4:69-89.

-6 Walley AJ, Asher JE, Froguel P: The genetic contribution to non-syndromic human obesity. Nat Rev Genet 2009;10:431-442.

7 Database Online Mendelian Inheritance in Man (OMIM AT): Obesity. http://omim.org/ (accessed January 2, 2014).

8 Kang JX: The coming of age of nutrigenetics and nutrigenomics. J Nutrigenet Nutrigenomics 2012;5(1):I-II.

-9 Kang JX: Identification of metabolic biomarkers for personalized nutrition. J Nutrigenet Nutrigenomics 2012; 5(2):I-II.

$\$ 10$ Marti A, Goyenechea E, Martínez JA: Nutrigenetics: a tool to provide personalized nutritional therapy to the obese. J Nutrigenet Nutrigenomics 2010;3:157-169.

11 Abete I, Navas-Carretero S, Marti A, Martinez JA: Nutrigenetics and nutrigenomics of caloric restriction. Prog Mol Biol Transl Sci 2012;108:323-346.

12 San Cristobal R, Milagro FI, Martínez JA: Future challenges and present ethical considerations in the use of personalized nutrition based on genetic advice. J Acad Nutr Diet 2013;113:1447-1454.

13 De Caterina R: Opportunities and challenges in nutrigenetics/nutrigenomics and health. J Nutrigenet Nutrigenomics 2010;3:137-143.

14 Bloss CS, Wineinger NE, Darst BF, Schork NJ, Topol EJ: Impact of direct-to-consumer genomic testing at long term follow-up. J Med Genet 2013;50:393-400.

15 Imai K, Kricka LJ, Fortina P: Concordance study of 3 direct-to-consumer genetic-testing services. Clin Chem 2011;57:518-521. 